\title{
Análisis de calidad del vino por medio de técnicas de inteligencia artificial
}

\author{
Luisa F. Galeano-Arias, Sergio G. Aguirre y Omar D. Castrillón-Gómez \\ Universidad Nacional de Colombia, Facultad de Ingeniería y Arquitectura, Departamento de Ingeniería \\ Industrial, Campus La Nubia Bloque Q piso 2, Manizales - Colombia. (correo-e: lufgaleanoar@unal.edu.co, \\ segilag@unal.edu.co, odcastrillong@unal.edu.co)
}

Recibido May. 26, 2020; Aceptado Jul. 27, 2020; Versión final Ago. 27, 2020, Publicado Feb. 2021

\begin{abstract}
Resumen
El objetivo de este trabajo es establecer por medio de técnicas de inteligencia artificial las variables más influyentes en la calidad sensorial del vino. Se analiza una variable dependiente (calidad) y 10 variables independientes: acidez volátil, ácido cítrico, azúcar residual, cloruros, dióxido de azufre libre, dióxido de azufre total, densidad, $\mathrm{pH}$, sulfatos, y alcohol. Se obtuvieron 300 registros de una base de datos y se seleccionaron estadísticamente las variables influyentes en un archivo. Este archivo es analizado con el algoritmo J48 (plataforma Weka) mediante un entrenamiento por medio de una validación cruzada. Los resultados muestran que, con una efectividad superior al 95\%, las variables más influyentes en la calidad del vino son: alcohol, pH, sulfatos, ácido cítrico y la relación alcohol y sulfatos. Se concluye que el control de estas 4 variables es suficiente para mejorar la calidad del vino. No obstante, es necesario ampliar estos estudios a un espectro muestral más amplio.
\end{abstract}

Palabras clave: inteligencia artificial; validación cruzada; calidad sensorial; vino; espectro muestral

\section{Wine quality analysis through artificial intelligence techniques}

\begin{abstract}
The objective of this research study is to determine the most influential variables in wine sensory quality by applying artificial intelligence techniques. The variables examined included one dependent variable (quality) and 10 independent variables: volatile acidity, citric acid, residual sugar, chlorides, total sulfur dioxide, density, $\mathrm{pH}$, sulfates, and alcohol. A total of 300 records were obtained from a database and the most influential variables were statistically selected to structure a file. This file is analyzed using the J48 algorithm (Weka Platform) by cross-validation training. The results show that, with over a 95\% effectiveness, the most influential variables in the quality of wine are: alcohol, $\mathrm{pH}$, sulfates, citric acid, and the proportion of alcohol and sulfates. It is concluded that controlling these 4 variables is sufficient to improve wine quality. However, it is necessary to conduct further research by expanding sampling spectrum.
\end{abstract}

Keywords: artificial intelligence; cross validation; wine sensory quality; sample spectrum 


\section{INTRODUCCIÓN}

Actualmente, se han establecido muchos mecanismos para determinar la calidad sensorial del vino, siendo en muchos casos subjetivos, con el respaldo de personas expertas (Catadores) o pruebas muy costosas (Portinale et al., 2017). En esta investigación se transforma un método subjetivo en un método objetivo, por medio de técnicas de minería de datos. Si bien existen algunos trabajos enfocados en estudiar objetivamente las características físico - químicas del vino y su influencia en la calidad sensorial (PájaroEscobar et al., 2019; Cortes et al., 2009), muchos otros trabajos han sido realizados de forma subjetiva. En este artículo se toma como referencia una base de datos de vinos encontrada en (Cortes et al., 2009), con el fin de determinar la calidad de los vinos, por medio de una técnica basada en minería de datos. Predecir la calidad del vino, de forma subjetiva, es un problema que implica tiempo y personal especializado en las vineras. Una estrategia Inteligente para resolver este problema, les permitirá a las empresas del sector, una mejor innovación, competitividad y calidad de vino. Aspecto, que puede afectar el estado de ánimo y emociones de los consumidores (Danner et al., 2016).

Algunos autores han analizado este problema desde aspectos geográficos, culturales, físicos, químicos, ambientales y bilógicos entre muchos otros factores. En Rinaldi et al., (2020), se estudian algunas técnicas para determinar la calidad del vino. En la anterior referencia se analiza la influencia del prensado y el área geográfica en la calidad del vino. En este proceso se estudian los parámetros de intensidad y sub- calidades de astringencia, sabor, olor, aroma por medio un jurado capacitado. En Sáenz-Navajas et al., (2014), se estudian los atributos extrínsecos, responsables de la calidad del vino, percibidos por los consumidores de dos regiones vineras en España y Francia. En Fayolle et al., (2019), se estudian los factores ambientales que controlan la calidad del vino, adaptando su producción a la variabilidad de las condiciones del suelo, espacio y condiciones ambientales. En Merin et al., (2018) se realiza un estudio sobre la influencia levadura pectinolítica Aureobasidium, en la calidad del vino. Por su parte en Cravero, (2019) se analiza la calidad y característica de los vinos orgánicos, analizando técnicas que permitan diferenciarlos.

En el mismo sentido del párrafo anterior, se enfocan los siguientes estudios: En Divita et al., (2019) se realiza un estudio sobre las percepciones de las percepciones de la calidad de los vinos, mediante un sistema de estimación de ecuaciones, encontrándose como cambian las motivaciones y determinantes en el consumo del vino. En Schelezki et al., (2020), se realiza un análisis químico combinado volátiles y perfiles sensoriales para determinar la calidad del vino. En Lock et al., (2019) se analiza la influencia de las ubicaciones y agrupaciones de las bodegas en la calidad del vino. En Charters y Pettigrew, (2007) se analizan las percepciones de los consumidores de vino sobre la calidad del mismo, como resultado se encuentran las dimensiones intrínsecas y extrínsecas, influyentes en la calidad del vino. En Towantakavanit et al., (2011) se hace un análisis de las cualidades de los vinos kiwi, determinando el contenido de azúcar, alcohol, acidez, $\mathrm{pH}$, color, fenoles totales y actividad antioxidante, con el fin de encontrar la mayor cantidad de características deseables para la producción del vino.

En un sentido diferente a los trabajos mencionados anteriormente, otros autores han propuesto métodos basados en técnicas de inteligencia artificial para analizar la calidad de los vinos. En Portinale et al., (2017) se emplean técnicas de aprendizaje automático, con el fin de realizar un análisis de datos químicos, para proteger los vinos más valiosos contra falsificaciones, estás técnicas de minería de datos empleadas, permiten realizar análisis de una forma más económica. Así mismo, en Khalafyan et al., (2019) se realiza un diseño de mezclas y superficies triangulares, de las cualidades sensoriales del vino rojo, con la ayuda de procesos estadísticos - informáticos, lográndose mejorar su calidad y mezcla. Por su parte, en Conde et al., (2017), se desarrolla un método robótico y de visión artificial para evaluar la calidad de los vinos, sustituyendo las evaluaciones sensoriales y subjetivas realizadas, por un proceso objetivo. En Petropoulos et al., (2017) se diseña una herramienta basada en la toma de dediciones de criterios múltiples por medio de la lógica difusa para determinar objetivamente la calidad del vino.

En el mimo sentido, en Brillante et al., (2015), se predicen las características físico químicas de las uvas, mediante técnicas de aprendizaje automático y análisis de textura, lográndose muy buenos resultados. En Buratti et al., (2007) se diseña una nariz y lengua electrónica las cuales por medio de un modelo de regresión basado en algoritmos genéticos establecen una predicción de los descriptores sensoriales del vino de una forma rápida y objetiva. Similarmente, en Rodríguez et al., (2019) se usa un sistema de nariz electrónico con la ayuda de una red neuronal y máquinas de soporte vectorial para detectar la calidad del vino. En Gupta, (2018) se realiza, por medio de técnicas de aprendizaje automático (regresión lineal, redes neuronales y máquinas de soporte vectorial), una selección de las características más importantes para predecir la calidad del vino. En Hu et al., (2018) se detecta de una forma rápida tres parámetros para la clasificación y calidad del vino: Espectroscopia infrarroja, selección de longitud de ondas mediante un algoritmo de colonia de hormigas, acompañado de un modelo de regresión de mínimos cuadrados. 
Las técnicas inteligentes también han sido usadas en la solución de diversos problemas relacionados. Existen diversos métodos (PCA, K-vecinos, Maquinas de soporte vectorial, árboles de clasificación y regresión, entre otras técnicas) con el fin de evaluar y determinar la calidad de los alimentos (JiménezCarvelo et al., 2019). En este mismo sentido se han empleado técnicas de espectroscopia (Ríos-reina et al., 2020). En Mihaljević et al., (2019), se mejora la calidad del vino, al mejorar las propiedades de la uva y logarse una mejor extracción en la materia colorante. Así mismo, en Da Costa et al., (2018) se realiza un proceso de reconocimiento geográfico de vinos, por medio de un proceso de aprendizaje automático y selección de características. Por su parte en Costa et al., (2019) se usa una máquina de soporte vectorial y una red neuronal para clasificar los vinos de algunos países suramericanos: Argentina, Brasil, Chile y Uruguay. En Urtubia et al., (2007) por medio de técnicas de minería de datos y Análisis de principales componentes se predicen los problemas anómalos que pueden existir en la fermentación del vino.

De otro lado, se resalta que actualmente existen diferentes técnicas para el desarrollo de análisis multivariado basadas en inteligencia artificial y otras técnicas: árboles de clasificación, reglas de inferencia, algoritmos evolutivos, redes artificiales, máquinas de aprendizaje automático, algoritmos de Clúster, algoritmos bayesianos, técnicas matemáticas y estadísticas entre muchas otras técnicas. Sin embargo, las técnicas de aprendizaje automático han cobrado gran relevancia hoy en día, dado que permiten construir modelos a partir de los datos deduciendo patrones configurados por ellos. En este sentido es posible clasificar las técnicas desarrolladas por la plataforma Weka (Witten et al., 2017), como técnicas de aprendizaje automático, los cuales generan mejores resultados cuando el número de datos empleados es mayor, aspecto que transforma la plataforma de aprendizaje y minería de datos Weka en una herramienta idónea para esta clase de análisis multivariado. Esta plataforma permite inferir el comportamiento de una variable dependiente con base en los valores que puedan tomar un conjunto de variables independientes, las cuales presenten una correlación moderada frente a la variable dependiente objeto de estudio (Bouckaert et al., 2013).

Esta investigación parte del análisis de una variable dependiente denominada calidad sensorial del vino (usualmente determinada por catadores cuyo criterio puede ser subjetivo), la cual es medida según 10 variables fisicoquímicas independientes: Acidez volátil, ácido cítrico, azúcar residual, cloruros, dióxido de azufre libre, dióxido de azufre total, densidad, $\mathrm{PH}$, sulfatos, alcohol. La medición de cada una de estas variables independientes juega un papel primordial en la calidad del vino (https://www.catadelvino.com): a) Acidez volátil. Esta variable hace referencia al ácido acético de un vivo e influye directamente en su calidad, cuanto menor es esta variable, mayor es la calidad del vino. b) Ácido cítrico. Produce el sabor ligeramente amargo que caracteriza los buenos vinos. c) Azúcar residual. Es el azúcar que queda después de fermentar el vino y tiene una gran influencia en la cata de mismo. Según su cantidad el vino puede ser seco, semiseco, semidulce y dulce. d) Cloruros. La elevación de los cloruros afecta la calidad del vino y la capacidad de las levaduras y bacterias de llevar a cabo la fermentación alcohólica y maloláctica, respectivamente (Donkin et al., 2010). e) Dióxido de azufre libre, total y Sulfatos. La oxidación del Dióxido de azufre consume el oxígeno y genera los sulfatos, teniendo la proporción de esta última variable gran influencia en la calidad del vino. f) Densidad. Es el espesor que se percibe en la boca cuando se cata un vino. g) $\mathrm{PH}$. Es uno de los factores predominantes en la acidez del vino, factor decisivo en la calidad del mismo (Tenorio M et al., 2014). h) Alcohol. El grado de alcohol es un factor importante en la calidad del vino siendo ideal que este se encuentre entre 10 y 14.

En consideración a lo anteriormente expresado surge así la siguiente pregunta de investigación: ¿Cómo diseñar una metodología basada en un análisis multivariado y técnicas de minería de datos, la cual permita identificar las principales variables físico - químicas que influyen en la identificación de la calidad sensorial del vino? Para responder esta pregunta, es necesario establecer en esta investigación las principales causas y relaciones que pueden influir en la calidad del vino. Como se expresó en el párrafo anterior, la calidad del vino, usualmente es determinada por catadores cuyo criterio puede ser subjetivo. Mediante un algoritmo que puede ser clasificado como una técnica inteligente, se determinará esta calidad.

Finalmente, este artículo es estructurado de la siguiente forma: a) Inicialmente, se expone la metodología empleada para abordar el problema objeto de estudio. b) posteriormente, en la sección de resultados se establecen las principales causas que influyen en la calidad del vino, con base en los resultados del algoritmo de clasificación J48. c) En la sección de discusiones, se compara este estudio con otras investigaciones similares encontrados en la literatura empleada. d) Subsiguientemente, se presentan las conclusiones derivadas de los resultados, así como una lista de las referencias utilizadas.

\section{MATERIALES Y MÉTODOS}

En el diseño y desarrollo de esta metodología, se emplea un algoritmo de clasificación (J48) basado en el algoritmo C4.5, empleado para generar arboles de decisión con base en grupo de datos de entrenamiento, 
el cual es ejecutado mediante la plataforma de aprendizaje automático y minería de datos denominada Weka, Esta plataforma es descrita en (Witten et al., 2017). Por medio de esta técnica, se predice de forma anticipada la calidad que puede tener un vino. Esta predicción se hace con base en 10 variables independientes (Cortes et al., 2009): Acidez volátil (AV), ácido cítrico $(A C)$, azúcar residual $(A R)$, cloruros $(C L)$, dióxido de azufre libre $(S O L)$, dióxido de azufre total $(S O T)$, densidad $(D), P H$, sulfatos $(S)$, alcohol $(A)$. Igualmente, se emplea una variable dependiente denominada calidad $(Q)$, la cual es medida en una escala de 1 a 10, siendo uno la calidad más baja y 10 la más alta. Basándose en estudios metodológicos previos (Castrillón et al., 2020a) y las consideraciones realizadas al final de la introducción, la metodología empleada en esta investigación, fue estructurada en cinco pasos, así: (a) Construcción de la base de datos; (b) Correlación de las variables; (c) Construcción del archivo Weka; (d) Elaboración del árbol de decisión (e) Identificación de las principales causas influyentes en la calidad del vino. f) Optimización del proceso. (Ver Tabla 1).

Tabla 1: Descripción de la metodología

\begin{tabular}{|c|c|c|}
\hline Paso & Nombre & Descripción \\
\hline 1 & $\begin{array}{l}\text { Construcción de la base } \\
\text { de datos }\end{array}$ & $\begin{array}{l}\text { Tomando como referencia la base de datos descrita en (Cortes et al., 2009), se } \\
\text { toma una muestra aleatoria de } 300 \text { registros, de cada una de las variables } \\
\text { independientes y la variable dependiente analizada }\end{array}$ \\
\hline 2 & Correlación de variables & $\begin{array}{l}\text { Con base en cada una de las variables seleccionadas en el paso 1, se calcula la } \\
\text { correlación entre cada una de las variables independientes y la variable } \\
\text { dependiente, con el fin de seleccionar aquellas variables independientes que } \\
\text { ejerzan una influencia moderada sobre la variable dependiente. Es decir, las } \\
\text { variables con una correlación muy alta o muy baja son descartadas. Las } \\
\text { variables con correlacionas bajas no tiene un aporte significativo, mientras que } \\
\text { las variables con una correlación muy alta básicamente contienen la misma } \\
\text { información que la variable dependiente. (Castrillón et al., 2020b) }\end{array}$ \\
\hline 3 & $\begin{array}{l}\text { Construcción archivo } \\
\text { Weka }\end{array}$ & $\begin{array}{l}\text { Con las variables independientes seleccionadas en el paso anterior, se diseña el } \\
\text { encabezado del archivo Arff para la plataforma Weka. Esta plataforma es } \\
\text { descrita en (Witten et al., 2017). Este encabezado contiene las variables } \\
\text { seleccionadas en el paso } 2 \text {. De forma similar se estructura el cuerpo del archivo } \\
\text {.Arff con el fin de complementar el archivo objeto de análisis mediante esta } \\
\text { plataforma. }\end{array}$ \\
\hline 4 & $\begin{array}{l}\text { Elaboración del árbol de } \\
\text { decisión }\end{array}$ & $\begin{array}{l}\text { El archivo .arff construido en el paso 3, se analiza por medio de un clasificador } \\
\text { mediante el algoritmo J48, con el fin de generar el respectivo árbol de decisión. } \\
\text { Estos clasificadores, permiten muy buenos resultados con pocos datos (Valencia } \\
\text { et al., 2015). Sin embargo, se toma una muestra bastante amplia con el fin } \\
\text { garantizar todo el proceso. }\end{array}$ \\
\hline 5 & $\begin{array}{l}\text { Identificación de las } \\
\text { principales causas } \\
\text { influyentes en la calidad } \\
\text { del vino }\end{array}$ & $\begin{array}{l}\text { El respectivo árbol de clasificación, que se obtiene con base en el algoritmo J48 } \\
\text { (plataforma weka), permite identificar las principales causas, influyentes en la } \\
\text { calidad del vino. Estas causas estarán representadas por los nodos del árbol que } \\
\text { se generen. }\end{array}$ \\
\hline 6 & Optimización del proceso & $\begin{array}{l}\text { Con el fin de optimizar los resultados del Paso } 5 \text {. Se toma cada una de las } \\
\text { variables independientes seleccionadas en el Paso } 2 \text {, (con las cuales se } \\
\text { construye el árbol de clasificación) con estas variables se calcula la respectiva } \\
\text { matriz de correlación. En esta matriz de correlación, se seleccionan las dos } \\
\text { variables independientes v1 y v2, con la correlación más baja entre sí. Con las } \\
\text { variables seleccionadas se calculada en el programa Weka una nueva variable } \\
\text { calculada igual a v2/v1. Finalmente se vuelve a generar un árbol de clasificación } \\
\text { el cuál incluya la nueva variable calculada y nuevamente se identifican las } \\
\text { principales causas influyentes en la calidad del vino. }\end{array}$ \\
\hline
\end{tabular}

\section{RESULTADOS}

Paso 1. Construcción de la base de datos. Como resultado del paso 1 de la metodología, la siguiente base de datos es construida. Ver Tabla 2 (Por su tamaño solo se muestra parte de la tabla):

Tabla 2: Base de datos generada.

\begin{tabular}{|l|l|l|l|l|l|l|l|l|l|l|}
\hline AV & AC & AR & CL & SOL & SOT & D & PH & S & Al & Q \\
\hline & & & & & & & & & & \\
\hline 0.27 & 0.36 & 20.7 & 0.05 & 45 & 170 & 1.00 & 3.00 & 0.45 & 8.80 & 6 \\
\hline 0.30 & 0.34 & 1.60 & 0.05 & 14 & 132 & 0.99 & 3.30 & 0.49 & 9.50 & 6 \\
\hline. &. &. &. &. &. &. &. &. &. &. \\
\hline. &. &. &. &. &. &. &. &. &. &. \\
\hline 0.23 & 0.32 & 8.50 & 0.06 & 47 & 186 & 1.00 & 3.19 & 0.40 & 9.90 & 6 \\
\hline
\end{tabular}


Paso 2. Correlación de variables. La correlación de cada una de las variables independientes, con la variable dependiente, es ilustrada en la Tabla 3 (Las variables de color gris presentan correlación moderada):

Tabla 3: Tabla de correlaciones.

\begin{tabular}{|l|l|l|l|l|l|l|l|l|l|l|}
\hline & AV & AC & AR & CL & SOL & SOT & D & PH & S & Al \\
\hline Q & 0.23 & 0.31 & 0.19 & 0.26 & 0.14 & 0.22 & 0.43 & 0.50 & 0.46 & 0.49 \\
\hline
\end{tabular}

Paso 3. Construcción archivo Weka. En la elaboración de este archivo, se tomaron las variables que presentaban una correlación moderada, respecto a la variable dependiente. Estas variables están sombreadas en la Tabla 3. El archivo Weka es estructurado en dos partes. La Tabla 4, muestra el encabezado, mientras que la Tabla 5 muestra el cuerpo del archivo (Por su tamaño solo se muestra parte del archivo).

Tabla 4: Encabezado del archivo Weka.

\begin{tabular}{|l|l|}
\hline Atributo & Tipo de datos \\
\hline @attribute Acido_citrico_AC NUMERIC & NUMERIC \\
\hline @attribute Cloridros_CL NUMERIC & NUMERIC \\
\hline @attribute Densidad_D NUMERIC & NUMERIC \\
\hline @attribute PH NUMERIC & NUMERIC \\
\hline @attribute Sulfatos_S NUMERIC & NUMERIC \\
\hline @attribute Alcohol_AI NUMERIC & NUMERIC \\
\hline @attribute Calidad_Q & $\{" 1 ", " 2 ", " 3 ", " 4 ", " 5 ", " 6 ", " 7 ", " 8 ", " 9 ", " 10 "\}$ \\
\hline
\end{tabular}

Tabla 5: Cuerpo del archivo.

\begin{tabular}{|c|c|c|c|c|c|c|}
\hline 0.36 & 0.05 & 1.00 & 3.00 & 0.45 & 8.80 & 6 \\
\hline 0.34 & 0.05 & 0.99 & 3.30 & 0.49 & 9.50 & 6 \\
\hline. &. &. &. &. &. &. \\
\hline 0.32 & 0.06 & 1.00 & 3.19 & 0.40 & 9.90 & 6 \\
\hline
\end{tabular}

Paso 4. Elaboración del árbol de decisión. Con base en el archivo anterior es posible predecir el comportamiento de la variable deseada, mediante el algoritmo de clasificación J48. Este algoritmo es interpretado por medio de la plataforma Weka, mediante un proceso de validación cruzada. La anterior interpretación permite generar el respectivo árbol de clasificación, el cual presenta una efectividad del $94 \%$. Los anteriores aspectos son ilustrados en Tabla 6 y la Figura 1.

Tabla 6: Porcentaje de clasificación

\begin{tabular}{|l|l|l|}
\hline Correctly Classified Instances & 284 & $94.67 \%$ \\
\hline Incorrectly Classified Instances & 16 & $5.33 \%$ \\
\hline Kappa statistic & 0.91 & \\
\hline Mean absolute error & 0.02 & \\
\hline Root mean squared error & 0.10 & \\
\hline Relative absolute error & $16.24 \%$ & \\
\hline Root relative squared error & $42.78 \%$ & \\
\hline
\end{tabular}

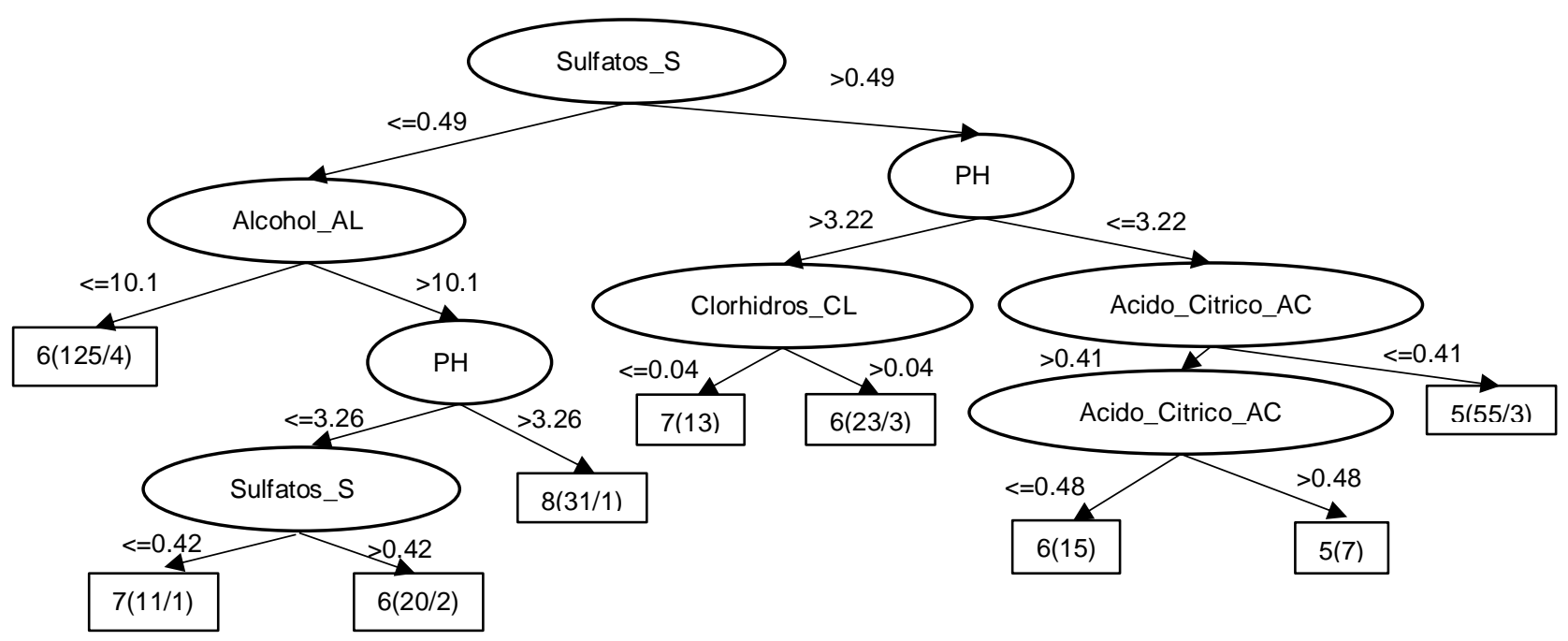

Fig. 1: Árbol de decisión. 
Paso 5. Identificación de las principales causas influyentes en la calidad del vino. Como se observa en el árbol de decisión (Figura 1), las variables más influyentes en la calidad del vino son. Sulfatos, Alcohol, PH y Cloridros. La interrelación de estas variables conduce a obtener vinos de calidades superiores: Sulfatos $(<=0.49) \rightarrow$ Alcohol $(>=10.1) \rightarrow \mathrm{PH}(>=3.26) \rightarrow$ 8; Sulfatos $(<=0.49) \rightarrow$ Alcohol $(>=10.1) \rightarrow \mathrm{PH}(<=3.26) \rightarrow$ Sulfatos $(<=0.42) \rightarrow 7$; Sulfatos $(>=0.49) \rightarrow \mathrm{PH}(>=3.22) \rightarrow$ Cloridros $(<=0.04) \rightarrow 7$.

Paso 6. Optimización del proceso. Como resultado de aplicar el Paso 6 de la metodología, en las variables independientes empleadas para construir el respectivo archivo .arff (Paso 3), se obtiene la siguiente matriz de correlaciones ver Tabla 7:

Tabla 7: Matriz Correlaciones. Variables seleccionadas

\begin{tabular}{|l|r|r|r|r|r|r|r|}
\hline Cor & \multicolumn{1}{l|}{ AC } & \multicolumn{1}{l|}{ CL } & D & \multicolumn{1}{l|}{ PH } & \multicolumn{1}{l}{ S } & \multicolumn{1}{l|}{ AL } & Q \\
\hline AC & 1.00 & -0.42 & -0.23 & 0.08 & 0.19 & 0.51 & 0.31 \\
\hline CL & -0.42 & 1.00 & 0.45 & 0.06 & -0.17 & -0.54 & -0.26 \\
\hline D & -0.23 & 0.45 & 1.00 & -0.26 & 0.07 & -0.86 & -0.43 \\
\hline PH & 0.08 & 0.06 & -0.26 & 1.00 & -0.22 & 0.27 & 0.50 \\
\hline S & 0.19 & -0.17 & 0.07 & -0.22 & 1.00 & 0.01 & -0.46 \\
\hline AL & 0.51 & -0.54 & -0.86 & 0.27 & 0.01 & 1.00 & 0.49 \\
\hline Q & 0.31 & -0.26 & -0.43 & 0.50 & -0.46 & 0.49 & 1.00 \\
\hline
\end{tabular}

En la Tabla 7, se muestra la correlación más baja, entre las variables independientes $\mathrm{AL}$ y $\mathrm{S}$, en consecuencia, la nueva variable calculada en Weka será definida como AL/S. Incluida esta variable en el nuevo árbol de clasificación, este se simplifica y el porcentaje de aciertos mejora, lográndose una clasificación superior al 95\%. Ver Tabla 8 y Figura 2:

Tabla 8: Proceso optimizado. Porcentaje de aciertos

\begin{tabular}{|l|l|l|}
\hline Correctly Classified Instances & 286 & $95.3333 \%$ \\
\hline Incorrectly Classified Instances & 14 & $4.6667 \%$ \\
\hline Kappa statistic & 0.9195 & \\
\hline Mean absolute error & 0.0167 & \\
\hline Root mean squared error & 0.096 & \\
\hline Relative absolute error & $13.9998 \%$ & \\
\hline Root relative squared error & $39.7112 \%$ & \\
\hline Total Number of Instances & 300 & \\
\hline
\end{tabular}



Fig. 2: Árbol de decisión optimizado. 
Así mismo, las tablas 9 y 10 muestran la precisión de los resultados obtenidos en la clasificación, para cada una de clases seleccionadas, siendo la clase de vino 7 la que tiene la menor precisión con el $88.5 \%$ y la clase de vino 8 la de mayor precisión con el 96.7\%, obteniéndose un promedio de 95.3\%. Igualmente, en el árbol de la Figura 2, se muestran las variables más influyentes: AL/S, PH, S, AC. Observándose que la relación entre estas variables conduce a vinos de muy buena calidad, como se ilustra en la tabla 11. Finalmente, en la Tabla 12 se compara la técnica de clasificación empleada frente a otras técnicas existentes. Las técnica Weka de clasificación de la Tabla 12 son encontradas en: https://weka.sourceforge.io/ y https://waikato.github.io/

Tabla 9: Matriz de precisión

\begin{tabular}{|c|c|c|c|c|c|c|}
\hline Positivos verdaderos & Falsos positivos & Precision & Recalculo & Medida F & Area ROC & Clase \\
\hline 0.896 & 0.013 & 0.952 & 0.896 & 0.923 & 0.921 & 5 \\
\hline 0.983 & 0.057 & 0.961 & 0.983 & 0.972 & 0.945 & 6 \\
\hline 0.920 & 0.011 & 0.885 & 0.92 & 0.902 & 0.924 & 7 \\
\hline 0.935 & 0.004 & 0.967 & 0.935 & 0.951 & 0.965 & 8 \\
\hline 0.953 & 0.038 & 0.953 & 0.953 & 0.953 & 0.940 & $\leftarrow$ Prom \\
\hline
\end{tabular}

Tabla 10: Matriz de confusión

\begin{tabular}{|r|r|r|r|l|}
\hline $\mathrm{a}$ & $\mathrm{b}$ & $\mathrm{c}$ & $\mathrm{d}$ & $\leftarrow$ Clasificación \\
\hline 60 & 6 & 1 & 0 & $\mathrm{a}=5$ \\
\hline 2 & 174 & 0 & 1 & $\mathrm{~b}=6$ \\
\hline 1 & 1 & 23 & 0 & $\mathrm{c}=7$ \\
\hline 0 & 0 & 2 & 29 & $\mathrm{~d}=8$ \\
\hline
\end{tabular}

Tabla 11: Variables relacionadas con la calidad del vino

\begin{tabular}{|c|c|c|c|c|}
\hline Var 1 & Var 2 & Var 3 & Var 4 & Var 5 \\
\hline $\mathrm{AL} / \mathrm{S} \rightarrow>26.67$ & $\rightarrow \mathrm{PH} \rightarrow>3.21$ & $\rightarrow 8$ & & \\
\hline $\mathrm{AL} / \mathrm{S} \rightarrow>26.67$ & $\rightarrow \mathrm{PH} \rightarrow<=3.21$ & $\rightarrow 7$ & & \\
\hline $\mathrm{AL} / \mathrm{S} \rightarrow>19 \mathrm{y}<=26.67$ & $\rightarrow \mathrm{PH} \rightarrow>3.47$ & $\rightarrow 7$ & & \\
\hline $\mathrm{AL} / \mathrm{S} \rightarrow>19 \mathrm{y}<=26.67$ & $\rightarrow \mathrm{PH} \rightarrow>3.45 \mathrm{y}<=3.47$ & $\rightarrow 8$ & & \\
\hline $\mathrm{AL} / \mathrm{S} \rightarrow>19 \mathrm{y}<=26.67$ & $\rightarrow \mathrm{PH} \rightarrow<=3.45$ & $\rightarrow \mathrm{S} \rightarrow>0.53$ & $\mathrm{AC} \rightarrow<=0.39 \rightarrow$ & $\rightarrow 7$ \\
\hline $\mathrm{AL} / \mathrm{S}<=19$ & $\rightarrow 7$ (Vinos de baja calidad) & & & \\
\hline
\end{tabular}

Tabla 12: Comparación con otras técnicas de clasificación.

\begin{tabular}{|l|l|l|}
\hline Técnica & Acierto & Descripción \\
\hline BFtree & $95.33 \%$ & Árbol de clasificación construido con base en la primera mejor decisión \\
\hline DecStum & $75 \%$ & Clasificación basada en una técnica de decisión stump \\
\hline FT & $94.67 \%$ & Árbol de aprendizaje funcional \\
\hline LMT & $95 \%$ & Árboles de modelo logístico \\
\hline NNBtree & $94.66 \%$ & Clasificador bayesiano Naives \\
\hline RanFor & $94.66 \%$ & Bosques aleatorios \\
\hline RandTre & $95 \%$ & Árbol aleatorio - con k atributos al azar elegidos de un nodo \\
\hline Reptree & $93.33 \%$ & Aprendizaje rápido de árboles de decisión \\
\hline SimpCar & $95.33 \%$ & Poda de mínimo costo de complejidad \\
\hline
\end{tabular}

\section{DISCUSIÓN}

En esta investigación se realiza un proceso para establecer la calidad de los vinos. Este proceso finalmente es reducido a la interacción de unas pocas variables $\mathrm{AL} / \mathrm{S}, \mathrm{PH}, \mathrm{S}, \mathrm{AC}$, resaltándose que si la relación $\mathrm{AL} / \mathrm{S}$, es menor que 19, el vino producido no será de buena calidad. En las diferentes referencias literarias relacionadas en este artículo no se encontró un proceso similar (basado en técnicas inteligentes), con un porcentaje de aciertos superior al 95\% ver: Petropoulos et al. (2017), Gupta, Y. (2018), Rodríguez et al. (2019), Hu et al. (2018), etc. Este hallazgo, les permitirá a las empresas relacionas con la industria del vino, establecer un control más efectivo para lograr producir vinos de mejor calidad. El control de las variables relacionadas en los resultados, permitirá lograr este aspecto. Respecto al PH este varía entre 3 y 4 (Tenorio $\mathrm{M}$ et al., 2014), siendo uno de los factores que determinan su acides. Los vinos tintos considerados tradicionalmente mejores que los blancos tiene una acides entre 3.3 y 3.6 un aspecto que puede ser ilustrado en la cuarta fila de la tabla 9. Por su parte el ácido cítrico (AC) produce ese sabor ligeramente amargo que tienen los buenos vinos.

Otras variables identificadas en este estudio como el alcohol $(A L)$ también encuentran una relevancia importante en la literatura (Tenorio $\mathrm{M}$ et al., 2014), siendo lo ideal para los vinos embotellados que esta, se 
encuentre entre 10 y 14 grados. Un aspecto ilustrado en la figura 1 donde se establece que los vinos de calidad superior deben tener una concentración de alcohol mayor a 10.1 grados. No obstante, el reto en este punto es mantener la temperatura en 20 grados centígrados, al momento de la fermentación, con el fin de que las levaduras alcancen su mayor grado de desarrollo. En forma análoga, la literatura muestra que los sulfatos (S) en la proporción que se muestra en la figura 1, es adecuada para los vinos de mejor calidad (Pájaro-Escobar et al., 2018) y tiene gran relevancia en la misma. La importancia de cada una de estas dos variables $A L$ y $S$ trae como consecuencia que su proporción, identificada en los resultados como (AL/S), tenga gran importancia en la calidad del vino.

$\mathrm{Si}$ bien este trabajo se realiza sobre una muestra, se resalta que la misma produce resultados estadísticamente válidos y mejores que otras técnicas similares (Tablas 9,10 y 12) encontradas en la literatura. Como se expresó en la metodología de este mismo artículo, los clasificadores (J48) producen muy buenos resultados con pocos datos, aspecto que facilita replicar la metodología propuesta en otras partes. Resaltándose que, si el viñero desea producir vinos de baja calidad o solo quiere evitar estos vinos, fundamentalmente debe mirar la relación existente entre las variables Alcohol (AL) y Sulfatos $(S)$ verificando que su razón sea menor o mayor que 19, según el efecto deseado. No obstante, como futuras líneas de investigación, se requiere un análisis sobre una población más amplia, con el fin de buscar generalizar los resultados y conclusiones encontrados en este artículo.

\section{CONCLUSIONES}

El análisis desarrollado en este trabajo, permite para un caso de estudio: a) establecer con una efectividad superior al 95\%, las variables más influyentes en la calidad de los vinos: Alcohol (AL), PH, sulfatos (S), ácidos cítricos (AC), encontrándose una especial relación entre las variables AL/S. b) Proponer una nueva metodología objetiva, basada en técnicas inteligentes, para determinar la calidad de un vino, por medio de datos numéricos, evitando costosos experimentos. c) Replicar este trabajo metodológico fácilmente en estudios similares. Para lograrlo solo es necesario estructurar y seguir la metodología paso a paso. d) Lograr una mayor validación de esta metodología, al permitir que la misma pueda ser fácilmente aplicada en diferentes vineros. No obstante, es importante ampliar el estudio a otros viñedos para una mayor generalidad de los resultados.

\section{AGRADECIMIENTOS}

Se agradece la colaboración a la Universidad Nacional de Colombia Sede Manizales, en especial al Departamento de Ingeniería Industrial. Igualmente se agradece al Center for Machine Learning and Intelligent Systems.

\section{REFERENCIAS}

Brillante, L., Gaiotti, F. y otros siete autores, Investigating the Use of Gradient Boosting Machine, Random Forest and their Ensemble to Predict Skin Flavonoid Content from Berry Physical-Mechanical Characteristics in Wine Grapes, http://dx.doi.org/10.1016/j.compag.2015.07.017, Computers and Electronics in Agriculture, 117 (2015), 186-193 (2015)

Bouckaert, R., Frank, E. y otros cinco autores, Weka Manual for Version 3-7-8, The University of Waikato, Hamilton, New Zeland (2013)

Buratti S., Ballabio, D. y otros dos autores, Prediction of Italian Red Wine Sensorial Descriptors from Electronic Nose, Electronic Tongue and Spectrophotometric Measurements by Means of Genetic Algorithm Regression Models, doi:10.1016/j.foodchem.2005.09.040, Food Chemistry, 100 , 211-218 (2007)

Castrillón, O., Sarache, W. y Ruiz, S., Predicción del Rendimiento Académico por medio de Técnicas de Inteligencia Artificial, http://dx.doi.org/10.4067/S0718-50062020000100093, Formación Universitaria, 13(1), 93-102 (2020a)

Castrillón, O., Sarache, W. y Ruiz, S., Predicción de las Principales Variables que conllevan al Abandono Estudiantil por medio de Técnicas de Minería de Datos, Formación Universitaria, 13(6), En prensa (2020b)

Charters, S. y Pettigrew, S., The Dimensions of Wine Quality, doi:10.1016/j.foodqual.2007.04.003, Food Quality and Preference, 18, 997-1007 (2007)

Conde, B., Fuentes, S. y otros cuatro autores, Development of a Robotic and Computer Vision Method to Assess Foam Quality in Sparkling Wines, http://dx.doi.org/10.1016/j.foodcont.2016.07.020, Food Control, 71, 383 e392 (2017)

Cortez, P., Cerdeira, A. y otros tres autores, Modeling Wine Preferences by Data Mining from Physicochemical Properties, https://doi.org/10.1016/j.dss.2009.05.016, In Decision Support Systems, 47(4), 547-553 (2009)

Costa, N., García, L. y otros dos autores, Using Support Vector Machines and Neural Networks to Classify Merlot Wines from South America, https://doi.org/10.1016/j.inpa.2018.10.003, Information Processing in Agriculture, 6, 265-278 (2019)

Cravero, M., Organic and Biodynamic Wines Quality and Characteristics: A Review, https://doi.org/10.1016/j.foodchem.2019.05.149, Food Chemistry, 295, 334-340 (2019) 
Da Costa, N., García, L. y otros tres autores, Geographical Recognition of Syrah Wines by Combining Feature Selection with Extreme Learning Machine, https://doi.org/10.1016/j.measurement.2018.01.052, Measurement, 120, 92-99 (2018)

Danner, L., Ristic, R. y otros cinco autores, Context and Wine Quality Effects on Consumers' Mood, Emotions, Liking and Willingness to Pay for Australian Shiraz Wines, http://dx.doi.org/10.1016/j.foodres.2016.08.006, Food Research International, 89, 254-265 (2016)

DiVita, G., Caracciol, F. y otros dos autores, Picking out a wine: Consumer Motivation Behind Different Quality Wines Choice, https://doi.org/10.1016/j.wep.2019.02.002, Wine Economics and Policy, 8, 16-27 (2019)

Donkin, R., Robinson, S. y otros cuatro autores, Sodium Chloride in Australian Grape Juice and Its Effect on Alcoholic and Malolactic Fermentation, American Journal of Enology and viticulture, ISSN: 0002-9254, 61, $392-400$ (2010)

Fayolle, E., Follain, S. y otros tres autores, Identification of Environmental Factors Controlling Wine Quality: A Case Study in Saint-Emilion Grand Cru Appellation, France, https://doi.org/10.1016/j.scitotenv.2019.133718, Science of the Total Environment, 694, 133718 (2019)

Gupta, Y., Selection of Important Features and Predicting Wine Quality Using Machine Learning Techniques, 10.1016/j.procs.2017.12.041, Procedia Computer Science, 125, 305-312 (2018)

Hu, L., Yin, C. y Zhimin, S., Rapid Detection of Three Quality Parameters and Classification of Wine Based on Vis-NIR Spectroscopy with Wavelength Selection by ACO and CARS Algorithms, https://doi.org/10.1016/j.saa.2018.07.054, Spectrochimica Acta Part A: Molecular and Biomolecular Spectroscopy, 205, 574-581 (2018)

Jiménez-Carvelo, A., González-Casado, A. y otros dos autores, Alternative Data Mining/Machine Learning Methods for the Analytical Evaluation of Food Quality and Authenticity - A Review, https://doi.org/10.1016/j.foodres.2019.03.063, Food Research International, 122, 25-39 (2019)

Khalafyan, A., Temerdashev, Z.A. y otros dos autores, Computer Analysis of the Sensory Qualities of Red Wines as a Method to Optimize their Blend Formulation, https://doi.org/10.1016/j.heliyon.2019.e01602, Heliyon, 5, e01602 (2019)

Lock, P., Mounter, S. y otros dos autores, Wineries and Wine Quality: The Influence of Location and Archetype in the Hunter Valley Region in Australia, https://doi.org/10.1016/j.wep.2019.10.002, Wine Economics and Policy, 8, 180 -190 (2019)

Merín, M. y Morata de Ambrosini, V., Kinetic and Metabolic Behaviour of the Pectinolytic Strain Aureobasidium Pullulans GM-R-22 During Pre-fermentative Cold Maceration and its Effect on Red Wine Quality, https://doi.org/10.1016/j.ijfoodmicro.2018.07.003, International Journal of Food Microbiology, 285, 18-26 (2018)

Mihaljević, M., Maslov-Bandić, L. y otros cuatro autores, Gamma Irradiation as Pre-Fermentative Method for Improving Wine Quality, https://doi.org/10.1016/j.Iwt.2018.11.016, LWT - Food Science and Technology, 101, 175-182 (2019)

Pájaro-Escobar, H., Benedetti, J. y García-Zapateiro, L., Caracterización Fisicoquímica y Microbiológica de un Vino de Frutas a base de Tamarindo (Tamarindus indica L.) y Carambola (Averrhoa carambola L.), http://dx.doi.org/10.4067/S0718-07642018000500123, Información Tecnológica, 29(5), 123-130 (2018)

Petropoulos, S., Spyridon, C. y otros cuatro autores, Fuzzy Logic Tool for Wine Quality Classification, https://doi.org/10.1016/j.compag.2017.11.015, Computers and Electronics in Agriculture, 142, 552-562 (2017)

Portinale, L., Leonardi, G. y otros cuatro autores, Authenticity Assessment and Protection of High-Quality NebbioloBased Italian Wines Through Machine Learning, https://doi.org/10.1016/j.chemolab.2017.10.012, Chemometrics and Intelligent Laboratory Systems, 171, 182-197 (2017)

Rinaldi, A., Louazil, P. y otros tres autores, Effect of Marc Pressing and Geographical Area on Sangiovese Wine Quality, https://doi.org/10.1016/j.Iwt.2019.108728, LWT - Food Science and Technology, 118, 108728 (2020)

Ríos-Reina, R., Azcarate, S.M. y otros dos autores, Assessment of UV-Visible Spectroscopy as a Useful Tool for Determining Grape-Must Caramel in High-Quality Wine and Balsamic Vinegars, https://doi.org/10.1016/j.foodchem.2020.126792, Food Chemistry, 323, 126792 (2020)

Rodriguez, J., Albarracin, E. y otros tres autores, Wine Quality Rapid Detection Using a Compact Electronic Nose System: Application Focused on Spoilage Thresholds by Acetic Acid, https://doi.org/10.1016/j.Iwt.2019.03.074, LWT Food Science and Technology, 108, 377-384 (2019)

Sáenz-Navajas, M-P., Ballester, J. y otros dos autores, Extrinsic Attributes Responsible for Red Wine Quality Perception: A Cross-Cultural Study Between France and Spain, http://dx.doi.org/10.1016/j.foodqual.2014.02.005, Food Quality and Preference, 35, 70-85 (2014)

Schelezki, O., Antalick, G. y otros dos autores, Pre-Fermentation Approaches to Producing Lower Alcohol Wines from Cabernet Sauvignon and Shiraz: Implications for Wine Quality Based on Chemical and Sensory Analysis, https://doi.org/10.1016/j.foodchem.2019.125698, Food Chemistry, 309, 125698 (2020)

Tenorio, M., Mateos-Aparicio, I. y otros seis autores, El Vino y su Análisis, Departamento de Nutrición y Bromatología II Facultad de Farmacia, Universidad Complutense, Madrid, España (2014)

Towantakavanit, K., Park, Y., Gorinstein, S., Quality Properties of Wine from Korean Kiwifruit New Cultivars, doi:10.1016/j.foodres.2011.01.028, Food Research International, 44, 1364-1372 (2011) 
Valencia, M., Correa, J. y Díaz, F., Métodos Estadísticos Clásicos y Bayesianos para el Pronóstico de Demanda. Un Análisis Comparativo, https://doi.org/10.15446/rev.fac.cienc.v4n1.49775, Revista Facultad de Ciencias Universidad Nacional de Colombia, 4(1), 52 -67 (2015)

Urtubia, A., Pérez-Correa, R. y otros dos autores, Using Data Mining Techniques to Predict Industrial Wine Problem Fermentations, doi:10.1016/j.foodcont.2006.09.010, Food Control, 18, 1512-1517 (2007)

Witten, I., Frank, E. y otros dos autores, Data Mining Practical Machine Learning Tools and Techniques, Morgan and Kaufman publication (Elsevier), ISBN-13: 978-0128042915, Cambrige, USA (2017) 